\title{
An Active Irradiation System with Automatic Beam Positioning and Focusing for a Medical Cyclotron
}

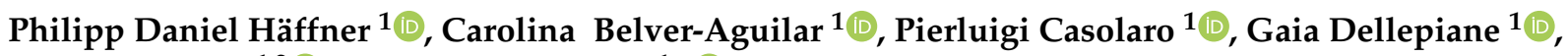 \\ Paola Scampoli ${ }^{1,2}$ (D) and Saverio Braccini ${ }^{1, *} *$ (D)
}

1 Albert Einstein Center for Fundamental Physics (AEC), Laboratory for High Energy Physics (LHEP), University of Bern, Sidlerstrasse 5, 3012 Bern, Switzerland; philipp.haeffner@lhep.unibe.ch (P.H.); carol.belver@gmail.com (C.B.-A.); pierluigi.casolaro@lhep.unibe.ch (P.C.); gaia.dellepiane@lhep.unibe.ch (G.D.); paola.scampoli@unina.it (P.S.)

2 Department of Physics "Ettore Pancini", University of Napoli Federico II, Complesso Universitariodi Monte S. Angelo, 80126 Napoli, Italy

* Correspondence: saverio.braccini@lhep.unibe.ch

check for updates

Citation: Häffner, P.D.; Belver-Aguilar, C.; Casolaro, P.; Dellepiane, G.; Scampoli, P.;

Braccini, $S$. An active irradiation system with automatic beam positioning and focusing for a medical cyclotron. Appl. Sci. 2021, 11, 2452. https://doi.org/10.3390/ app11062452

Academic Editor: Emilio Martines

Received: 17 February 2021

Accepted: 5 March 2021

Published: 10 March 2021

Publisher's Note: MDPI stays neutral with regard to jurisdictional claims in published maps and institutional affiliations.

Copyright: (c) 2021 by the authors. Licensee MDPI, Basel, Switzerland. This article is an open access article distributed under the terms and conditions of the Creative Commons Attribution (CC BY) license (https:// creativecommons.org/licenses/by/ $4.0 /)$.

\begin{abstract}
A novel active focusing system was developed for enhancing the irradiation performance of the $18 \mathrm{MeV}$ medical cyclotron in operation at the Bern University Hospital in view of the production of non-conventional medical radioisotopes using solid targets. In several cases, such as the production of ${ }^{43} \mathrm{Sc}$ and ${ }^{44} \mathrm{Sc}$, the beam has to be kept stable within a very small target of about $5 \mathrm{~mm}$ diameter. For this purpose, we conceived and realized an apparatus based on a compact focusing and steering magnet system followed by a two-dimensional beam monitoring detector and a specific feedback software that drives the magnet to optimize the beam for a given irradiation set-up. We report on the design, realization and validation beam tests performed using the research beam transfer line of the Bern cyclotron. We demonstrated that the beam spot can be kept on target thanks to the fact that the system automatically reacts to perturbations. Compactness is one of the key features of this system, allowing its use in accelerator facilities with limited space, such as medical cyclotrons for radioisotope production.
\end{abstract}

Keywords: particle accelerator; medical cyclotron; automatic irradiation system; beam diagnostics; solid targets; medical radioisotope production

\section{Introduction}

Compact medical cyclotrons for the production of radioisotopes for Positron Emission Tomography (PET) are common instruments in modern medicine. They accelerate $\mathrm{H}^{-}$ions at energies in the range 10-25 MeV with maximum currents in the range 100-300 $\mu \mathrm{A}$ [1]. Radioisotopes are usually produced using liquid targets, as in the case of ${ }^{18} \mathrm{~F}$, which is the most common radionuclide for PET imaging. Research is nowadays concentrating on the production of novel medical radionuclides which can be used to label bio-molecules. In particular, pairs of radiometals, one positron emitter for PET imaging and one $\beta^{-}$ emitter for therapy, can be used to label proteins and peptides paving the way to the new field of theranostics in nuclear medicine [2,3]. This is the case of the ${ }^{43} \mathrm{Sc} /{ }^{47} \mathrm{Sc}$ and ${ }^{44} \mathrm{Sc} /{ }^{47} \mathrm{Sc}$ pairs [4-7]. The production of novel radioisotopes with adequate purity and quantity using compact medical cyclotrons is challenging. A very promising method is the use of solid targets. The bombardment of isotope enriched materials in solid form allows production of activities in the 1-100 GBq range. Since the target can be made thin, an accurate knowledge of the beam energy and of the production cross section allows optimization of the radionuclide purity. Furthermore, targets are often necessarily small (about $5 \mathrm{~mm}$ diameter) as materials are expensive and available only in small quantities and in form of powder. In this framework, two key issues have to be addressedthe preparation of the target and the optimization of the irradiation process. As far as the 
target is concerned, our group developed a specific capsule that can fit a commercial Solid Target Station (STS) [8]. As far as the irradiation is concerned, STSs are usually mounted in out-ports of compact medical cyclotrons right after the stripper without any optical device for beam steering and focalization. This results in a beam of 10-15 mm Full Width at Half Maximum (FWHM) on target, which implies that only a small fraction ( $25 \%$ or less) of the beam hits the about $5 \mathrm{~mm}$ target material. The remaining part of the beam causes unwanted activation of the materials (mostly the capsule) and unnecessary stress for the accelerator. Furthermore, beam tuning procedures are very limited since the centering of the beam on target can only be performed in a coarse way by varying the angle of the stripper.

The irradiation procedures for the production of non-conventional radioisotopes using commercial STSs at compact medical cyclotrons need therefore to be optimized by the development of new equipment and methodologies, as reported for example in References $[9,10]$. However, it has to be considered that hospital-based cyclotron facilities consist of a single bunker and that very limited space is available to insert beam optics elements to focus and steer the beam. For this purpose, we conceived and developed an Automatic Focusing System (AFS) that steers and focuses the beam on target, controls its size and position and corrects its characteristics if necessary. It is based on a compact (about $1 \mathrm{~m}$ long) apparatus combining a magnet with both focusing and steering capabilities, a non-destructive two-dimensional beam monitoring detector and a specific feedback software control system.

The development of such an apparatus is complex and requires easy access to the beam area, which is not possible in the compact medical cyclotrons used for routine radioisotope production due to radiation protection issues. The medical cyclotron facility in operation at the Bern University Hospital (Inselspital) [11] was conceived to perform radioisotope production together with multi-disciplinary research by means of a $6 \mathrm{~m}$ long Beam Transfer Line (BTL) ending in a bunker with an independent access [12]. In this paper, we present the conception and realization of the AFS together with the first beam tests performed using the BTL. This work is fundamental in view of the installation of the AFS in the cyclotron vault to be used together with the STS for our investigations on novel radioisotopes for theranostics.

\section{Materials and Methods}

The AFS was developed to enhance the irradiation performance of the Bern medical cyclotron laboratory. This facility is based on an IBA 18/18 HC cyclotron providing proton beams with a nominal energy of $18 \mathrm{MeV}$ and beam currents in the range from a few $\mathrm{pA}$ to $150 \mu \mathrm{A}$ [13]. The cyclotron features eight out-ports: four are used for routine production of ${ }^{18} \mathrm{~F}$ for PET imaging, 1 is connected with a commercial IBA Nirta solid target station, two are reserved for future developments and one is connected to the BTL. The BTL is $6 \mathrm{~m}$ long and its optical elements consist of two horizontal-vertical $(\mathrm{H}-\mathrm{V})$ quadrupole doublets, the former located in the cyclotron bunker and the latter in a second bunker, where experiments are conducted [12].

The two hardware components of the AFS are-the Mini-PET Beamline (MBL) [14], and a two-dimensional beam profiler (UniBEaM). The MBL is an innovative compact (about $50 \mathrm{~cm}$ long) magnet embedding two quadrupole and two steering magnets within the same structure, produced by the Canadian company D-Pace. In order to maximize its compactness, the two quadrupoles are located next to each other without any drift space in between. The two bending dipole coils are enclosed within the quadrupoles, leading to interference effects. This unusual design allows focusing and steering the beam in a very limited space, as in a standard cyclotron bunker. The UniBEaM detector [15] was developed by our group to measure the beam profile in a wide range of currents. It is based on scintillating silica doped fibers passing through the beam. The position of the fiber is recorded together with its light output to obtain the beam profile at a given location along the beam line. Several commercial versions of the UniBEaM are produced under licence by D-Pace [16]. For the tests described in this paper, the AFS was installed 
at the end of the BTL, as shown in Figure 1. According to the specific measurement to be performed, three different instruments were used to assess the beam characteristics in the exact position where the IBA Nirta production solid target station is foreseen to be locateda two-dimensional UniBEaM profiler (dual axis UniBEaM by D-Pace), a two-dimensional non-destructive beam monitor (named $\pi^{2}$, indicated with (4) in Figure 1) followed by a beam dump or a specific target station with current read-out. The $\pi^{2}$ detector [17] is based on a thin (about $1 \mu \mathrm{m}$ ) aluminum foil coated by P47 scintillating material read-out by a Charge-Coupled Device (CCD) camera allowing to assess the beam characteristics at the position of the target in two dimensions. The foil is tilted by $45^{\circ}$ with respect to the horizontal plane. To measure the real dimensions of the beam, images are corrected by taking into account the effects due to the tilt and to distortions of the optics of the CCD camera.

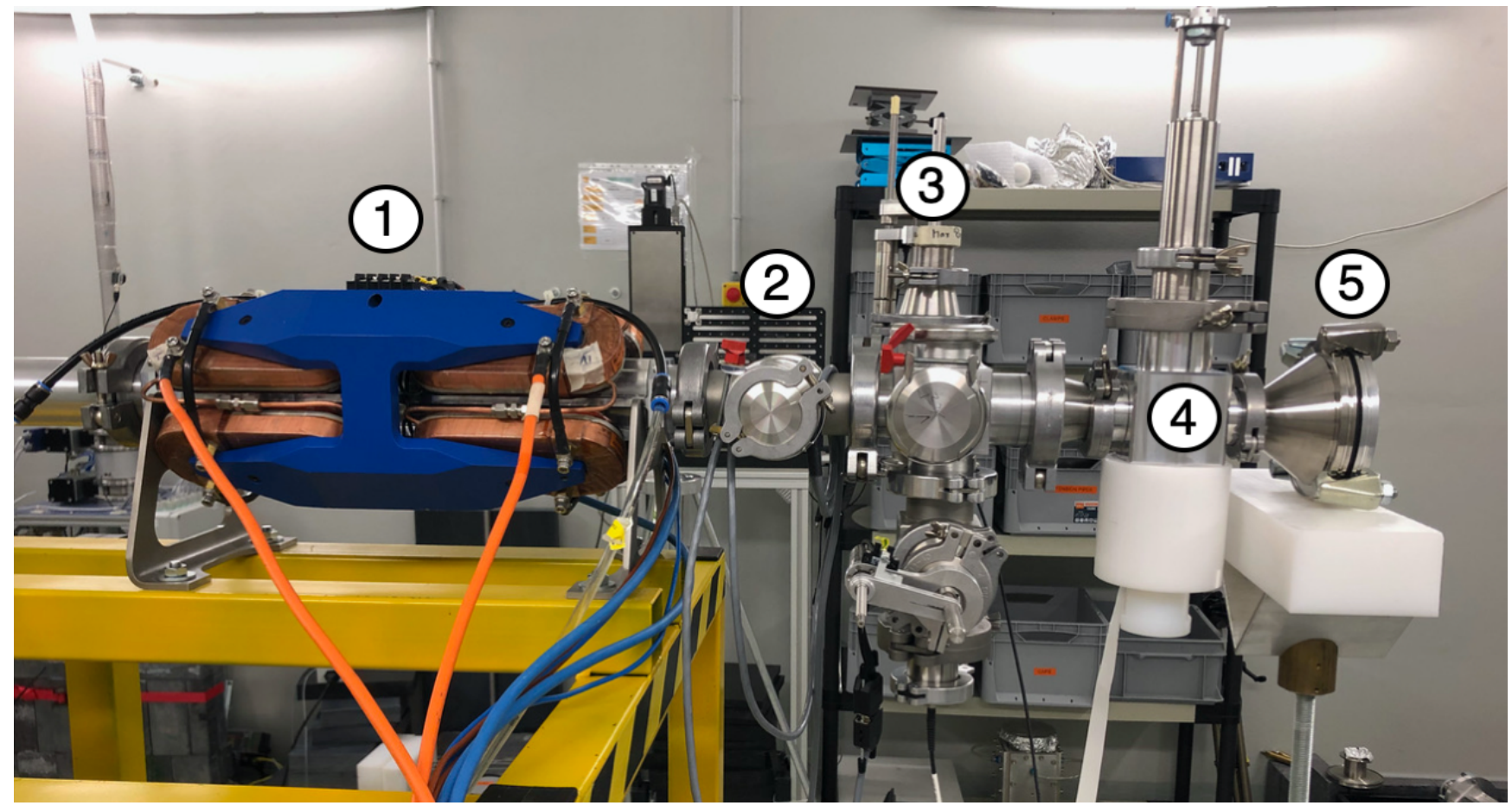

Figure 1. The Automatic Focusing System (AFS) under test in the Beam Transfer Line (BTL) bunker: (1) the Mini-PET Beamline (MBL); (2) drift space; (3) two-dimensional beam profile detector (UniBEaM); (4) $\pi^{2}$ detector (or, alternatively, two-dimensional UniBEaM detector or target station with current read-out); (5) beam dump.

The target station with current read-out was developed by our group to measure nuclear reaction cross sections [5]. It has a $6 \mathrm{~mm}$ diameter collimator behind which a specifically designed target coin [8] can be placed to perform production tests at low current (below $1 \mu \mathrm{A}$ ) since this device is not water-cooled. This coin has a pocket for the target material that is covered by a thin aluminum foil that produces a negligible degradation of the beam energy. Aluminum disks can be located in front of the coin to reduce the impinging energy if necessary. To suppress secondary electrons that can increase and therefore alter the current reading, an electrode was used as in Faraday cups (50 V bias voltage).

For the AFS, a specific two-dimensional UniBEaM detector ((3) in Figure 1) was developed, as shown in Figure 2. It features two silica fibers moving vertically and horizontally whose planes are only $5 \mathrm{~mm}$ apart. This is realized by a specific vacuum chamber, named Magic-Box, in which the KF-50 flanges are two-by-two appropriately staggered. The Magic-Box allows measuring the horizontal and the vertical profiles in two very close locations along the beam path. 

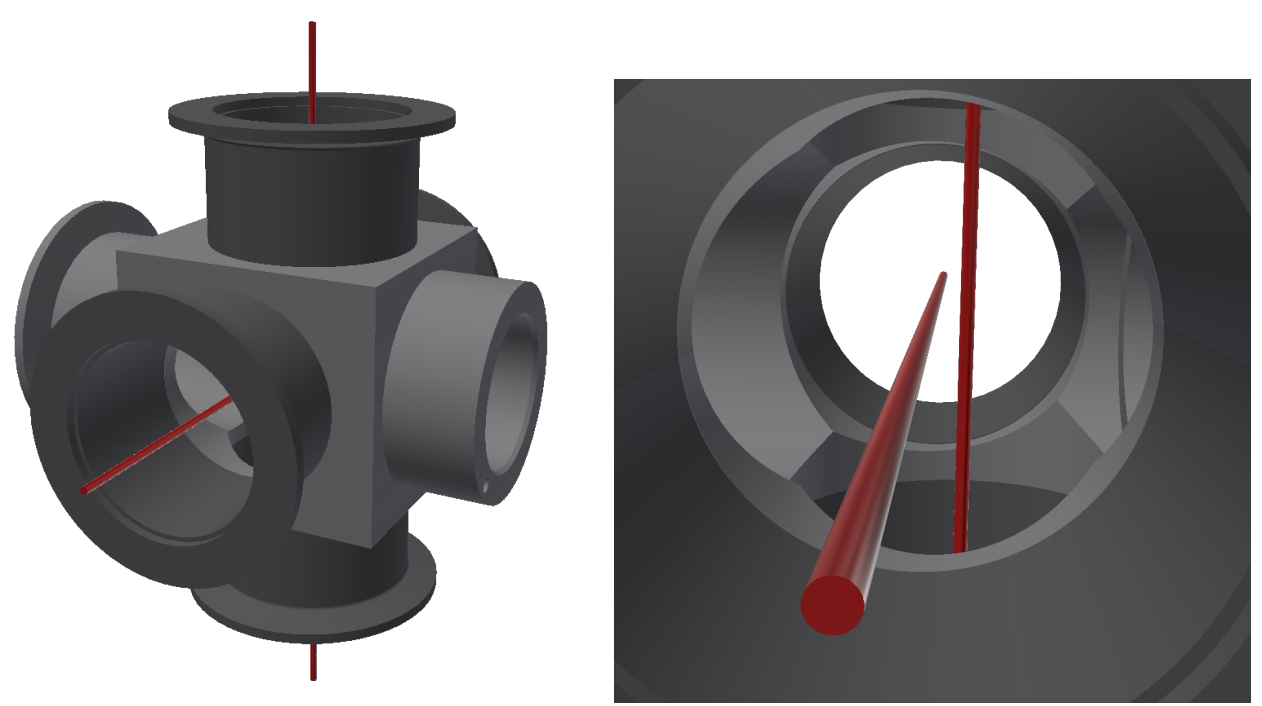

Figure 2. Computer-Aided Design (CAD) drawing of the Magic-Box that allows to operate two one-dimensional UniBEaM detectors (horizontal and vertical) and features five KF-50 flanges and a specific connection for the solid target station (left). Particular of the sensing fibers (drawn in red) (right). The distance between the planes in which the fibers move is $5 \mathrm{~mm}$.

The block scheme of the experimental set-up is reported in Figure 3, where the AFS is highlighted in red. The control system is a specific software based on a feedback algorithm. The beam profiles are measured by the UniBEaM and, if necessary, they are optimized to obtain the desired beam on target by varying the current in the power supplies of the MBL. The power supplies, provided by the Italian company CAEN-ELS , are of two kindsbipolar $( \pm 10 \mathrm{~A})$ for the steering magnets (FAST-PS 1020-200) and monopolar (0-100 A) for the quadrupoles (FAST-PS-M 7508). To avoid overheating in the MBL, the maximum current for the quadrupoles is limited to $75 \mathrm{~A}$. The currents in the magnets of the MBL are indicated as $\mathrm{I}_{x}$ and $\mathrm{I}_{y}$ for the two steering and $\mathrm{I}_{Q 1}$ and $\mathrm{I}_{\mathrm{Q} 2}$ for the downstream and upstream quadrupoles, respectively.
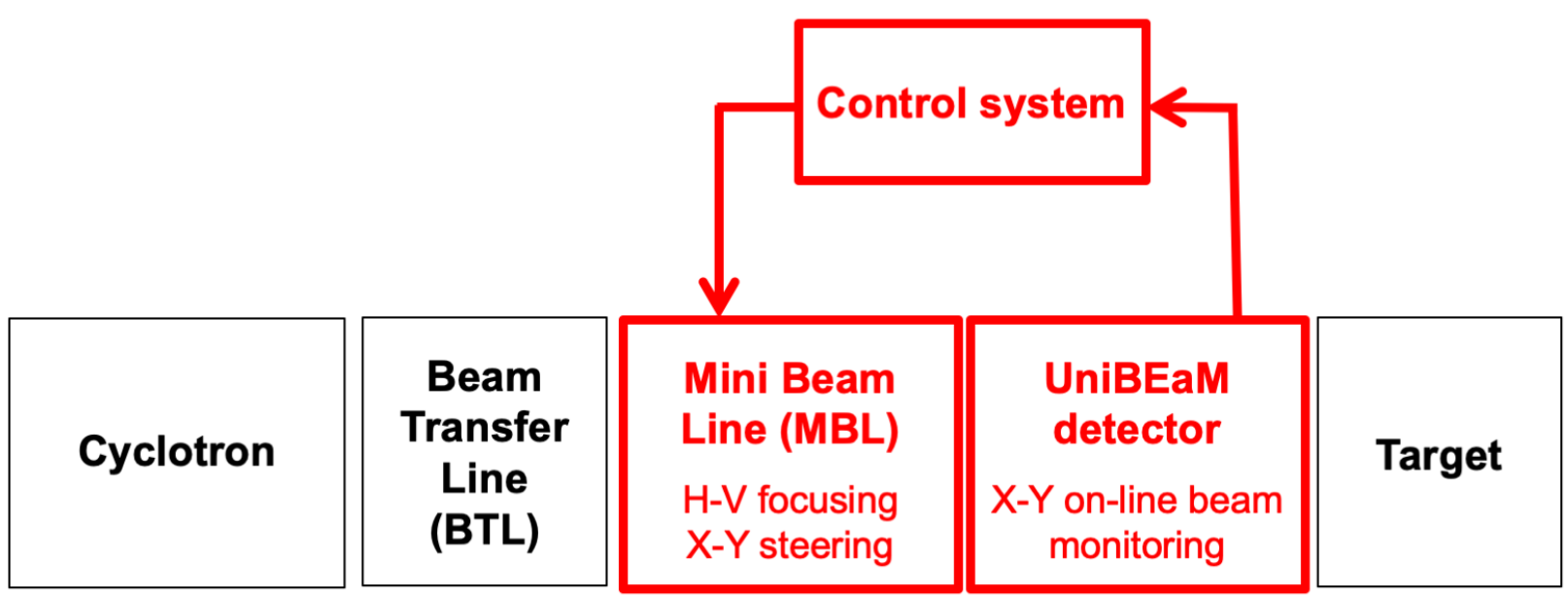

Figure 3. Scheme of the experimental set-up in which the Automatic Focusing System (AFS) is highlighted in red.

The control system (written in C++ running on Linux) is based on an optimisation algorithm aimed at focusing and steering the beam to irradiate the target region, as defined by the user. The only inputs of the algorithm are the horizontal and vertical profiles acquired by the UniBEam ((3) in Figure 1). In the ideal case, the beam should be fully contained in the target region, where it should feature a flat distribution. A centered but 
too narrow beam has to be avoided since it may produce overheating and a low production yield. The desired region is defined as two spatial ranges in the target plane, one in $x$ and one in $y$, where $z$ is the beam axis. Once the $x$ and $y$ profiles are acquired, the algorithm assigns an optimisation parameter to each of them. It is defined as the product of two subparameters - the ratio of the beam intensity in the target region over the overall intensity and the portion of the Full Width at Half Maximum (FWHM) of the beam contained in the target region over the full length of the target region. This second sub-parameter aims at obtaining an almost flat distribution. The higher the optimisation parameter, the better the beam profile is adapted to the target. If, following a scan, the optimisation parameter is found to be lower than a predefined threshold, the AFS starts the optimisation procedure. On the other hand, if the parameter is higher than the threshold, the system waits for the next scan. The time interval between successive scans is defined by the user. At the start of an irradiation, the user sets the MBL parameters according to predefined values which were previously experimentally determined, for example by locating a beam monitoring detector, as the $\pi^{2}$ or the UniBEaM, exactly at the location of the target. Once the beam deviates from the initial configuration and the profiles give an optimization parameter lower than the threshold, the optimization procedure is triggered. It acts on one of the four currents at the time and their order is defined by the user. Usually, $\mathrm{I}_{\mathrm{Q} 1}$ and $\mathrm{I}_{\mathrm{Q} 2}$ are first optimized, followed by $\mathrm{I}_{x}$ and $\mathrm{I}_{y}$. As soon as the optimization parameter gets over the threshold again, the procedure stops. The optimization algorithm proceeds in steps. At the first step, the selected magnet current is varied around the starting point by adding and subtracting a coarse amount (typically $\pm 1 \mathrm{~A}$ ). This allows to individuate the right direction for optimization. The current corresponding to the highest value of the optimization parameter is retained for the second step when two further variations are applied, whose size depends on the outcome of the first step. Beam profiles are measured at each successive step and, at the end of the procedure, the applied variations become smaller and smaller down to a user defined minimum. At each step, two variations are applied. They are allowed to be both positive, both negative or one negative and one positive. The choice is made on the basis of the previous step. Figure 4 shows two examples of the optimization procedure, where the steps are highlighted. It has to be noted that the optimization parameter as a function of the magnet currents was found to be continuous, as needed for an optimization procedure.
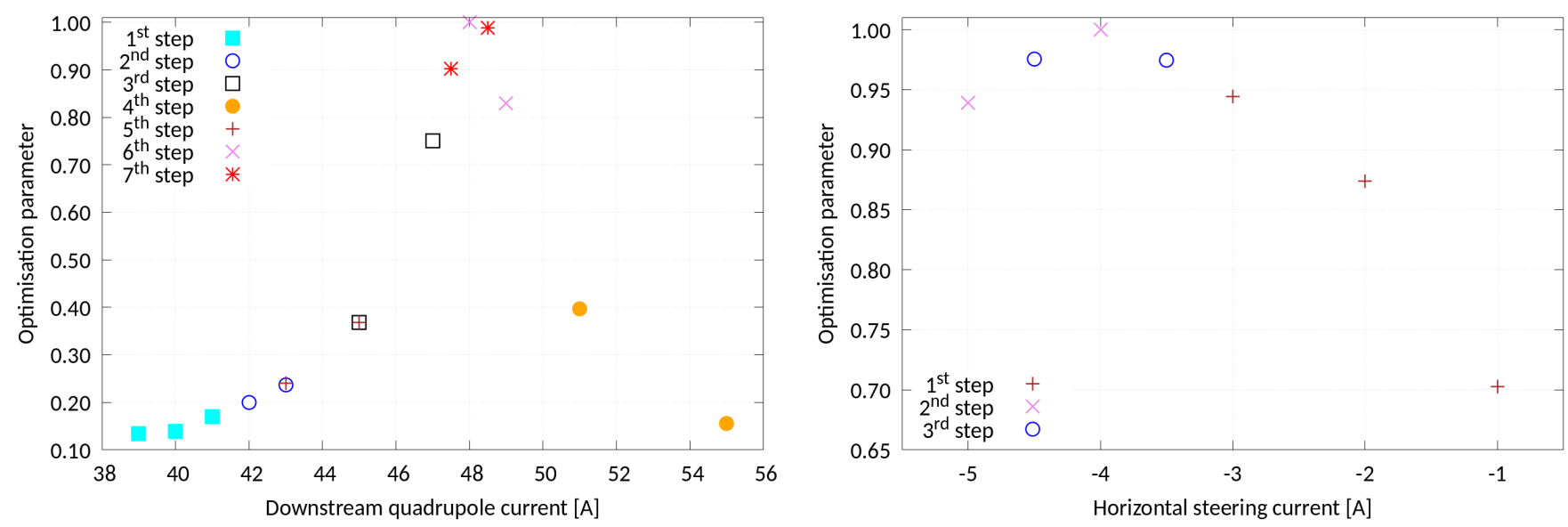

Figure 4. Examples of the optimization process for one quadrupole (left) and one steering magnet (right). The succession of the steps with the corresponding magnet current variations is highlighted.

The AFS optimizes the beam on the basis of the measurement of the beam profiles performed by the two-dimensional UniBEaM detector located about $30 \mathrm{~cm}$ upstream with respect to the target. It is therefore necessary to predict the size and position of the beam on target by means of calculations based on the Twiss parameters $\alpha, \beta$ and $\gamma$, which are functions of the beam along the MBL. The Twiss parameters were assessed by measuring 
the variation of the beam profile as a function of the gradient of the downstream quadrupole magnet, as described in Ref. [18], where the beam dynamics of the BTL was studied in detail. To estimate the size of the beam on target, we introduce a scaling factor $f$ as the ratio between the standard deviation of the beam at the position of the UniBEaM $\left(\sigma_{U B}\right)$ and on target $\left(\sigma_{\text {target }}\right)$. Using beam dynamics equations, one gets

$$
f=\frac{\sigma_{\text {target }}}{\sigma_{U B}}=\sqrt{L^{2} \cdot \frac{\gamma_{U B}}{\beta_{U B}}-2 \cdot L \cdot \frac{\alpha_{U B}}{\beta_{U B}}+1},
$$

where $L$ represents the distance between the UniBEaM and the target and the Twiss parameters are evaluated at the position of the UniBEaM. As far as the position is concerned, the horizontal shift $\Delta x$ of the beam profile centroid between the UniBEaM and the target can be estimated for small angles by using the formula:

$$
\Delta x=\frac{q\left(\int B(l) d l\right) c}{\sqrt{E_{k i n}^{2}+2 E_{k i n} m c^{2}}} \cdot L,
$$

where $q$ is the elementary electric charge, $c$ the speed of light, $B(l)$ the magnetic field profile within the MBL along the beam axis and $d l$ an infinitesimal element along this line. $B(l)$ was accurately measured by means of a Hall probe. Finally, $E_{k i n}$ is the kinetic energy of the proton and $m$ its mass. An analogous function is used for the vertical shift $\Delta y$. Equation (2) can be derived from the equations of motion of a charged particle in a magnetic field. A typical value of $f$ is 0.1 , which means that the beam is 10 times smaller on target with respect to what is measured by the UniBEaM. For $\Delta x$ and $\Delta y$ the correction is of a few millimeters.

\section{Results}

To predict the position and the shape of the beam on target, an accurate knowledge of the magnetic field produced by the MBL is essential. For this purpose, we performed a measurement campaign using a Hall probe. Effects due to magnetic hysteresis were studied in detail in order to implement the necessary corrections in the optimization algorithm.

Since the MBL embeds two quadrupole doublets and two steering dipoles within the same structure, overlapping effects occur that influence the bending and focusing capabilities. As an example, the effect of the quadrupole field on the dipole is shown in Figure 5. It can be noted that a quadrupole current of $50 \mathrm{~A}$ reduces the dipole field strength of about $20 \%$. The fit corresponds to an arc-tangent function, which is used along with the upstream profiles measured by the UniBEaM and Equations (1) and (2) to calculate the position and shape of the beam on target.

To verify the capability of predicting the shape of the beam on target, a UniBEaM detector was installed at the target position and the measured profiles were compared with the calculated ones. An example is shown in Figure 6, demonstrating a very good agreement.

A first series of tests of the AFS were performed to obtain a very focused beam on target starting with a broad beam. To get the smallest possible beam spot on target, we set the target region to $1 \mathrm{~mm}$ in $x$ and $1 \mathrm{~mm}$ in $y$. For this purpose, a $\pi^{2}$ detector was placed at the target location to visualize and assess the beam size (Figure 1). As shown in Figure 7, a very broad beam is measured before starting the AFS procedure that is able to focus it into the requested small spot. This procedure was successfully repeated for several initial beam conditions. It is important to remark that, even starting from a worse case situation (broad beam), a spot much smaller than the one required for the production of novel radioisotopes (about $5 \mathrm{~mm}$ diameter) can be obtained. 


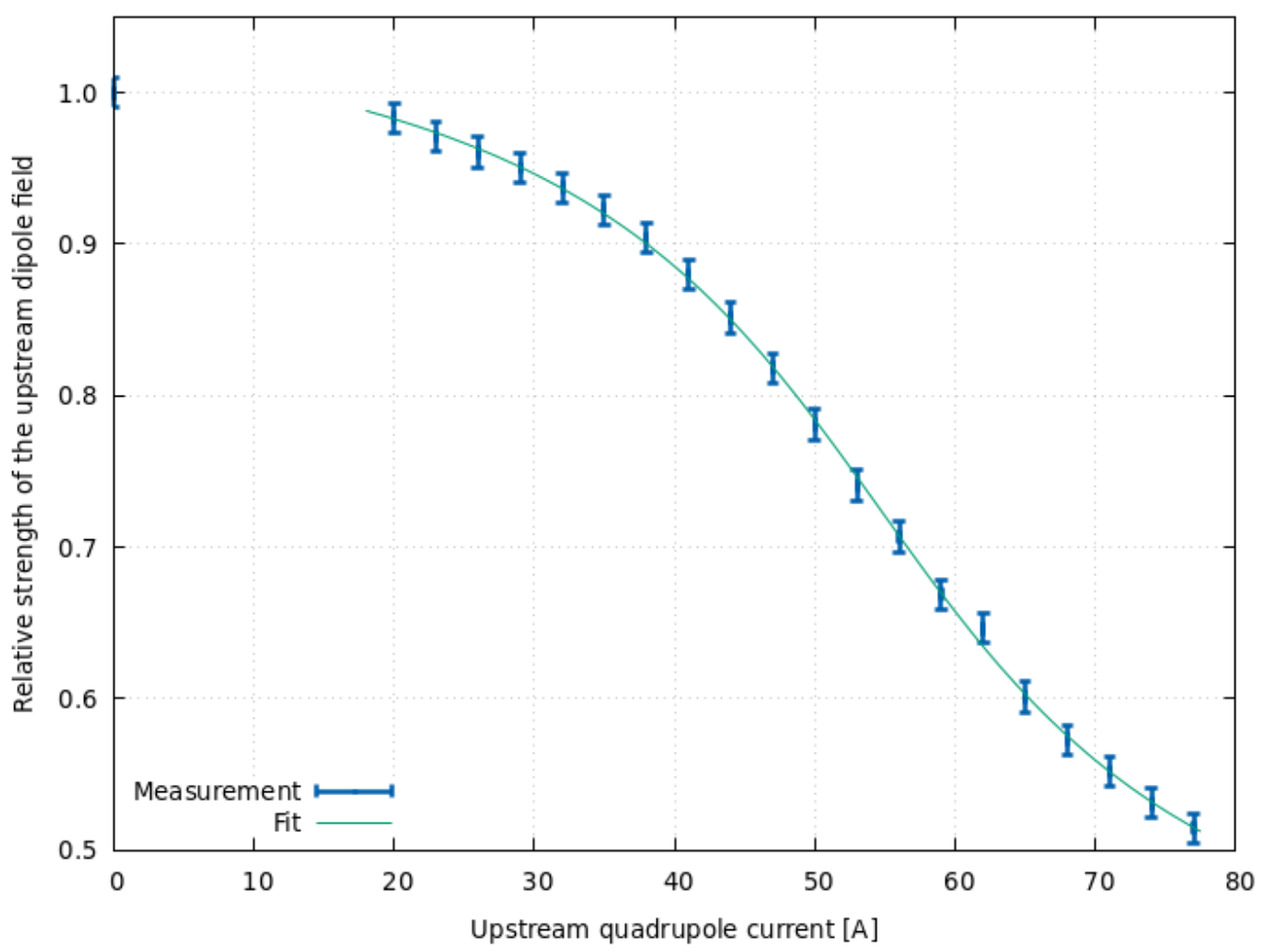

Figure 5. Relative strength of the upstream bending dipole as a function of the current in the upstream quadrupole.

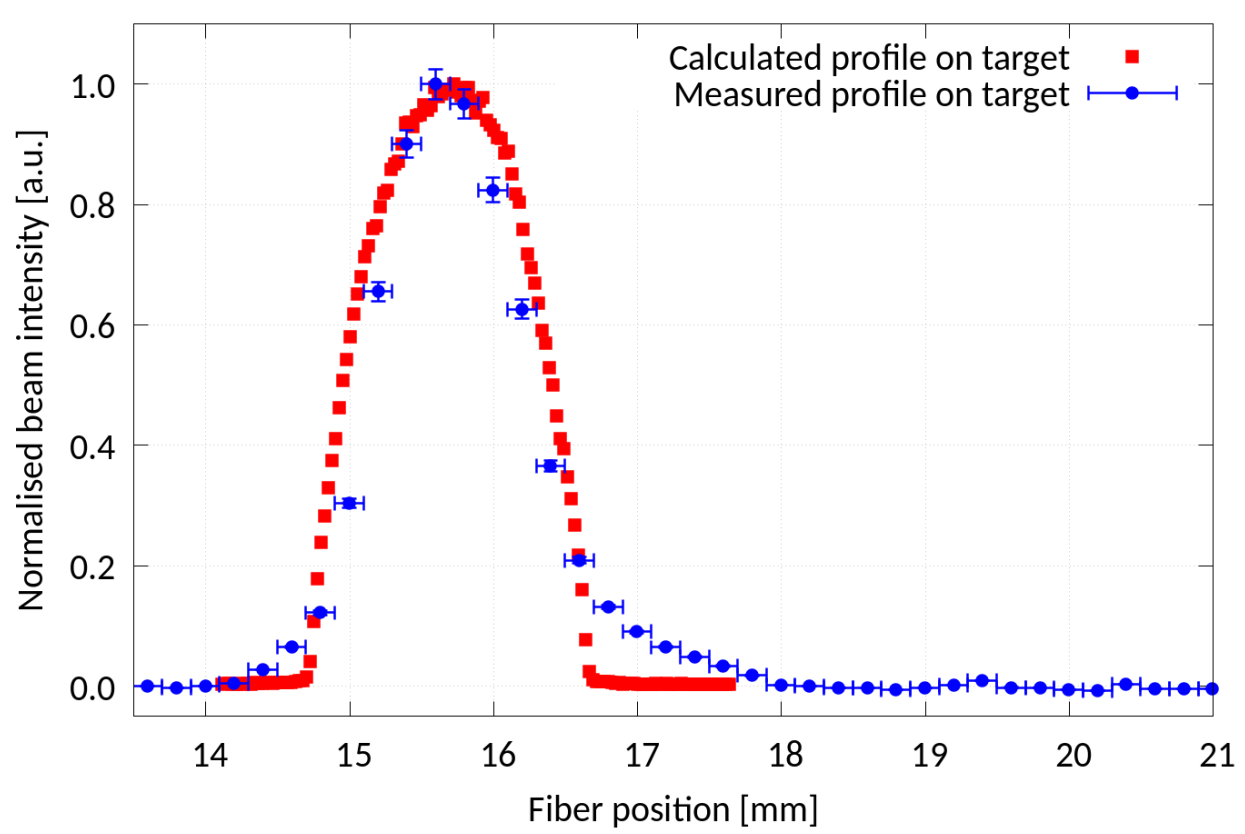

Figure 6. Beam profiles at the target position measured by the UniBEaM detector (blue) and calculated (red). 

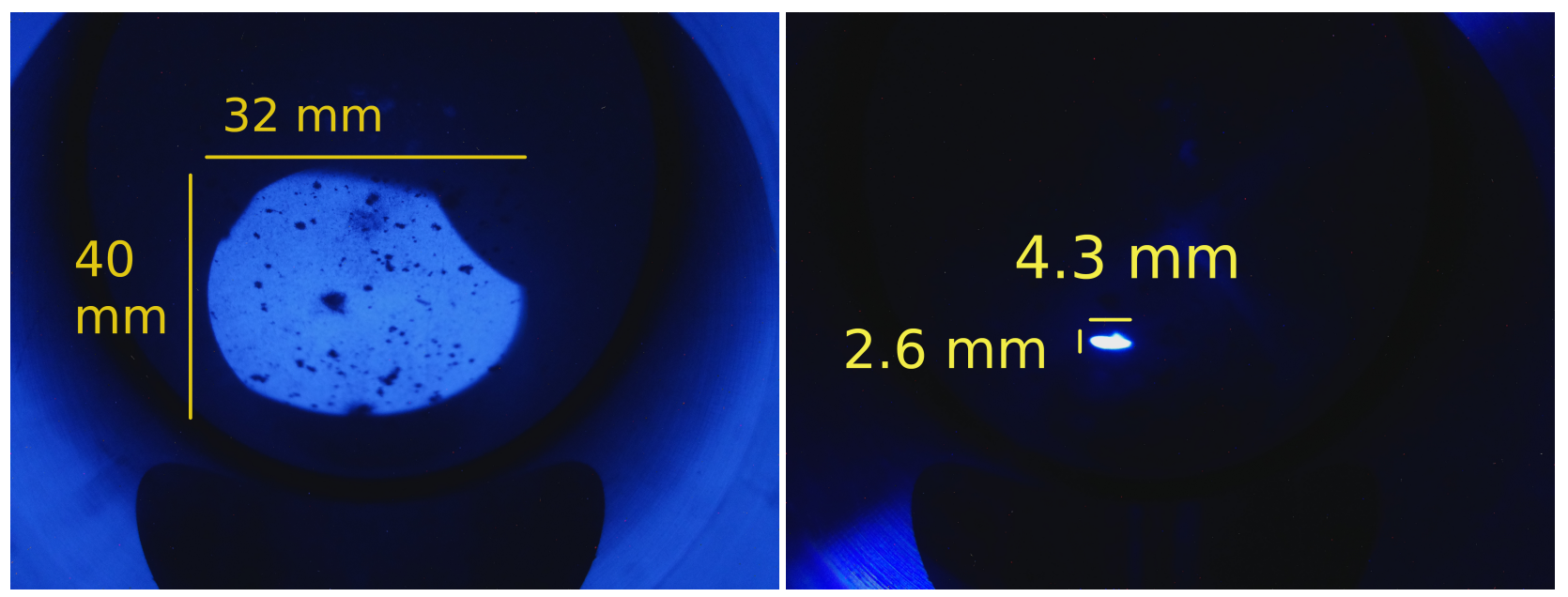

Figure 7. The two-dimensional profile of the beam measured with the $\pi^{2}$ detector before (left) and after (right) the optimisation procedure performed by the AFS. The real dimension of the beam is reported, once the effect of the vertical tilt of the scintillating foil is corrected for. The dark spots in the left figure are due to inhomogeneities in the coating of the scintillating foil.

A further test aims at evaluating the response of the AFS to an external perturbation of the beam that was produced by the horizontal and vertical steering magnets of the BTL, which are located upstream of the AFS. Figure 8 shows the beam profiles in the horizontal and vertical planes measured by a two-dimensional UniBEaM detector located at the position of the target. The measured profiles demonstrate the successful recovery of the beam position, width and intensity.
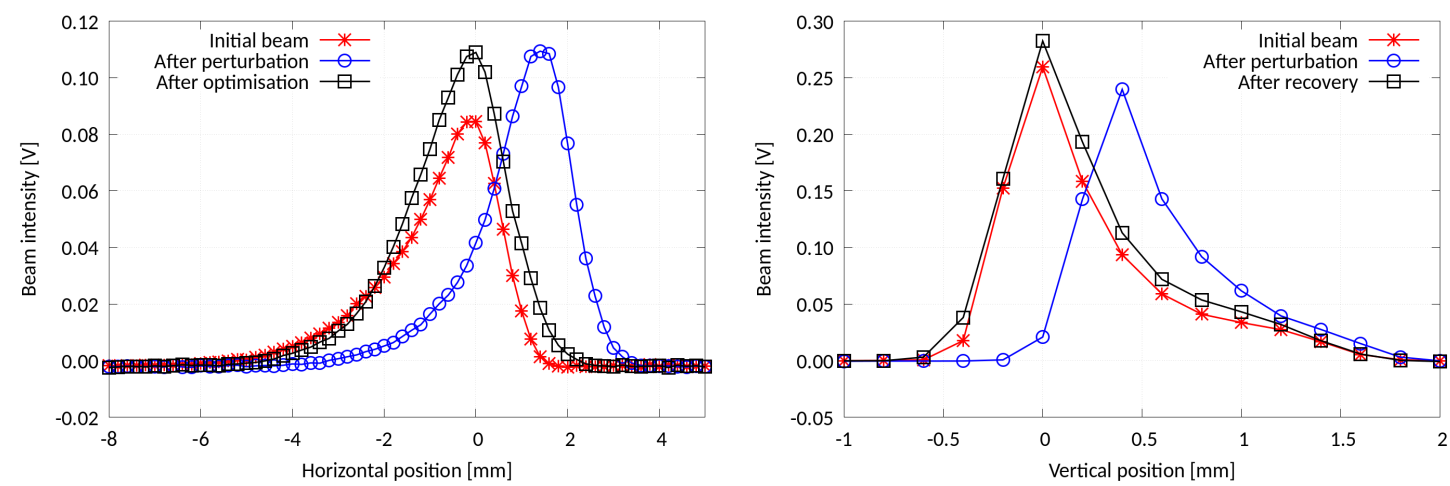

Figure 8. Capability of the AFS to restore the initial beam conditions after a perturbation. Horizontal (left) and vertical (right) beam profiles measured by a two-dimensional UniBEaM located at the position of the target. The output of the photo-diode of the commercial UniBEaM is reported in Volt.

For the production of radioisotopes, the current hitting of the target material is a key parameter. To test the performance of the AFS to recover the beam current on an about $5 \mathrm{~mm}$ diameter target following a perturbation, a beam dump with current read-out equipped with a $6 \mathrm{~mm}$ diameter collimator was installed at the target position. The current on target as a function of time is shown in Figure 9 when a $\sim 60 \%$ reduction of the beam current is produced by means of the steering magnets of the BTL. One can observe the evolution of the current on target all along the optimization procedure that brings to about $90 \%$ of the initial value. A full recovery was not possible since about $10 \%$ of the current was lost before the MBL. This was proved by measuring the current at the entrance of the MBL before and after the perturbation.

Analogously, Figure 10 shows the optimization procedure after a perturbation induced by acting on the quadrupoles of the BTL. In this case, a $\sim 30 \%$ reduction in the beam current was completely recovered. 


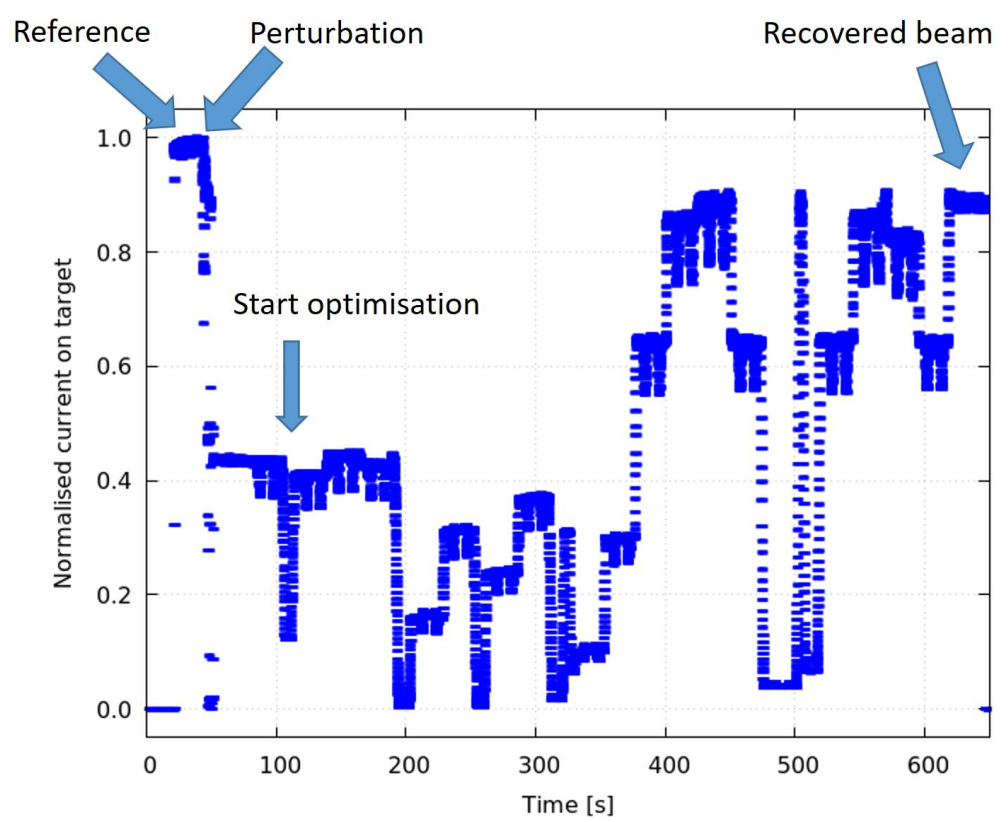

Figure 9. Beam current on target as a function of time during an optimization procedure when a perturbation is applied by means of the steering magnets of the BTL. The arrows indicate the phases of the procedure.

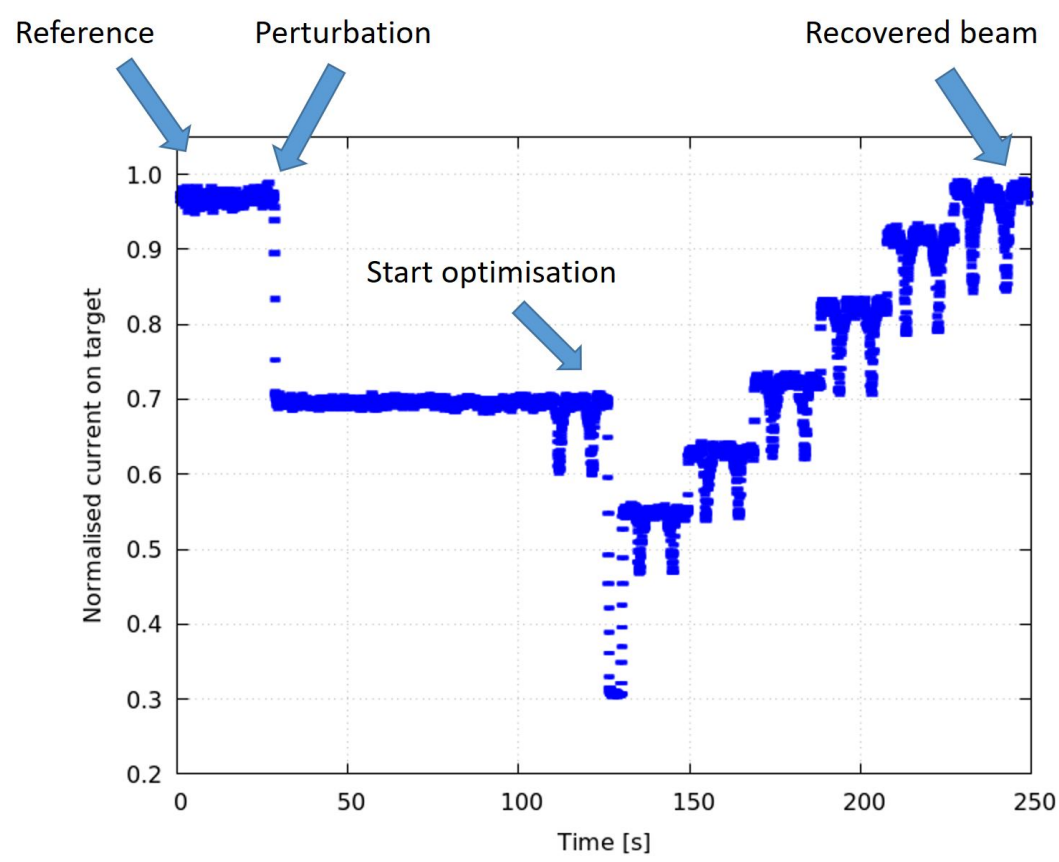

Figure 10. Beam current on target as a function of time during an optimization procedure when a perturbation is applied by means of the quadrupoles of the BTL. The arrows indicate the phases of the procedure.

The two examples reported above represent worse cases that will not occur during radioisotope production once the AFS is installed in the cyclotron bunker with the STS. In this case, perturbations will be much smaller and mostly due to drifts of the cyclotron magnetic field caused by temperature increase in the main coil. We are therefore confident that the AFS will be capable to correct the possible perturbations during irradiation with the STS. 
To test the enhancement of the performance elicited by the AFS in the production of radioisotopes using solid targets, we irradiated two natural zinc targets one with a broad and one with a focused beam, as in Figure 7. The targets had a diameter of $6 \mathrm{~mm}$ and a mass of $89 \pm 10 \mu \mathrm{g}$ and $87 \pm 10 \mu \mathrm{g}$, respectively. A target of the same dimensions but made of enriched material is used for the production of ${ }^{68} \mathrm{Ga}$ at the Bern cyclotron [8]. Since the target station at the end of the BTL is not water-cooled, the irradiation was limited to 2 min with currents of 0.92 and $17.5 \mathrm{nA}$ on target, with the flat and focused beam, respectively. In the first case, the flat beam represents a worse case situation since the beam extracted by the cyclotron has approximately $12 \mathrm{~mm}$ FWHM on target in a standard STS. On the other hand, the focused beam is such that all the protons hit the target and, since it is smaller than the target, it represents also an extreme case. For both measurements, we used the same settings for the cyclotron and for the BTL.

The produced specific activities of ${ }^{66} \mathrm{Ga}$ and ${ }^{67} \mathrm{Ga}$ at End of Bombardment (EoB) were measured by gamma spectroscopy with a High Purity Germanium (HPGe) detector (gamma lines at $1039 \mathrm{keV}$ and $93 \mathrm{keV}$, respectively). The results are reported in Table 1. The specific activity and the total charge on target are measured using the methodology we developed to assess radioisotope production cross sections [5]. The gain factor in the produced specific activities was found to be about 20. This is in line with the ratio of the current on target measured during irradiation. Once the AFS is installed in front of the STS in the cyclotron bunker, we expect a considerable increase of the production yield of which this extreme case represents an upper limit.

Table 1. Comparison of gallium production using natural zinc targets with a flat and an optimized beam.

\begin{tabular}{cccc}
\hline & $\begin{array}{c}\text { Produced Specific Activity } \\
\mathbf{6 6}_{\mathbf{G a}}\left[\frac{\mathbf{M B q}}{g}\right]\end{array}$ & $\begin{array}{c}\text { Produced Specific Activity } \\
\mathbf{6 7}_{\mathbf{G a}}\left[\frac{\mathbf{M B q}}{g}\right]\end{array}$ & $\begin{array}{c}\text { Total Charge on Target } \\
{[\mathbf{n C}]}\end{array}$ \\
\hline Flat beam & $53 \pm 7$ & $(1.9 \pm 0.3) \times 10^{-3}$ & $106.0 \pm 0.4$ \\
\hline Focused beam & $1180 \pm 140$ & $(3.9 \pm 0.6) \times 10^{-2}$ & $2074 \pm 8$ \\
\hline Gain factor & $\mathbf{2 2} \pm \mathbf{4}$ & $\mathbf{2 1} \pm \mathbf{4}$ & $\mathbf{1 9 . 6} \pm \mathbf{0 . 1}$ \\
\hline
\end{tabular}

\section{Conclusions}

In the framework of the research program on radioisotopes for theranostics ongoing at the Bern medical cyclotron laboratory, a novel compact automatic focusing system (AFS) was conceived, realized and tested. It aims at enhancing the production performance using solid target stations. This apparatus is capable of focusing the beam on small targets down to $5 \mathrm{~mm}$ diameter or less and to keep it in position, thanks to a feedback algorithm that, based on the measurements of beam profiles, acts on a combined compact focusing and steering magnet, named Mini-PET Beamline (MBL). Beam tests were successfully carried out by installing the AFS at the end of the $6 \mathrm{~m}$ long beam transfer line (BTL). We demonstrated that the system is able to react to perturbations induced by changing the beam characteristics at the input of the MBL by acting on the focusing and steering elements of the BTL, assuring the convergence of the MBL coil currents towards an optimal radioisotope production. This optimization procedure minimizes the activation of the materials outside the target, thus optimizing radiation protection issues, transport procedure and use of the accelerator. For the same amount of extracted current, a gain factor of about 20 was found by comparing the worse case of a flat beam with an automatically optimized focused beam.

On the basis of these promising results, the installation of the AFS in the bunker of the Bern cyclotron is planned, where the solid target station (STS) is in operation. Being about $1 \mathrm{~m}$ long, the AFS can be easily adapted to any existing medical cyclotron facility in view of investigations and applications of new radioisotopes for theranostics in nuclear medicine. 
Author Contributions: S.B. conceived the system; S.B. and P.H. contributed to its design and construction together with the LHEP mechanical workshop; P.H., C.B.-A. and P.C. contributed to the installation of the system in the BTL; P.H. developed and implemented the automatic focusing feedback algorithm; P.H., P.C., C.B.-A. and G.D. performed the beam tests; P.H., C.B.A, P.C., G.D., P.S. and S.B. contributed to the data analysis; S.B., P.S., P.H. and P.C. wrote the paper; P.H., C.B.-A., P.C., G.D., P.S. and S.B. revised the paper; S.B. coordinated the project. All authors have read and agreed to the published version of the manuscript.

Funding: This research was partially funded by the Swiss National Science Foundation (SNSF). Grants: 200021_175749 and CRSII5_180352.

Acknowledgments: We acknowledge contributions from the LHEP engineering and technical staff who helped us with the mechanical and electronics challenges during the prototype development and installation. We thank the SWAN Isotopen AG maintenance team for the collaboration.

Conflicts of Interest: The authors declare no conflict of interest.

\section{References}

1. Braccini, S. Compact medical cyclotrons and their use for radioisotope production and multi-disciplinary research. In Proceedings of the Cyclotrons'16, Zurich, Switzerland, 11-16 September 2016; pp. 229-234. [CrossRef]

2. Yordanova, A.; Eppard, E.; Kürpig, S.; Bundschuh, R.A.; Schönberger, S.; Gonzalez-Carmona, M.; Feldmann, G.; Ahmadzadehfar, H.; Essler, M. Theranostics in nuclear medicine practice. OncoTargets Ther. 2017, 10, 4821-4828. [CrossRef] [PubMed]

3. Langbein, T.; Weber, W.A.; Eiber, M. Future of Theranostics: An Outlook on Precision Oncology in Nuclear Medicine. J. Nucl. Med. 2019, 9, 13S-19S. [CrossRef] [PubMed]

4. Huclier-Markai, S.; Alliot, C.; Kerdjoudj, R.; Mougin-Degraef, M.; Chouin, N.; Haddad, F. Promising Scandium Radionuclides for Nuclear Medicine: A Review on the Production and Chemistry up to In Vivo Proofs of Concept. Cancer Biother. Radiopharm. 2018, 33, 316-329. [CrossRef] [PubMed]

5. Carzaniga, T.S.; Auger, M.; Braccini, S.; Bunka, M.; Ereditato, A.; Nesteruk, K.P.; Scampoli, P.; Türler, A.; Van der Meulen, N. Measurement of ${ }^{43} \mathrm{Sc}$ and ${ }^{44} \mathrm{Sc}$ production cross-section with an $18 \mathrm{MeV}$ medical PET cyclotron. Appl. Radiat. Isot. 2017, 129, 96-102. [CrossRef] [PubMed]

6. Carzaniga, T.S.; Braccini, S. Cross-section measurement of ${ }^{44 m} \mathrm{Sc},{ }^{47} \mathrm{Sc},{ }^{48} \mathrm{Sc}$ and ${ }^{47} \mathrm{Ca}$ for an optimized ${ }^{47} \mathrm{Sc}$ production with an $18 \mathrm{MeV}$ medical PET cyclotron. Appl. Radiat. Isot. 2019, 143, 18-23. [CrossRef] [PubMed]

7. van der Meulen, N.P.; Hasler, R.; Talip, Z.; Grundler, P.V.; Favaretto, C.; Umbricht, C.A.; Müller, C.; Dellepiane, G.; Carzaniga, T.S.; Braccini, S. Developments toward the Implementation of ${ }^{44}$ Sc Production at a Medical Cyclotron. Molecules 2020, 25, 4706. [CrossRef] [PubMed]

8. Braccini, S.; Belver-Aguilar, C.; Carzaniga, T.S.; Dellepiane, G.; Häffner, P.D.; Scampoli, P. Novel Irradiation Methods for Theranostic Radioisotope Production with Solid Targets at the Bern Medical Cyclotron. In Proceedings of the International Conference on Cyclotrons and their Applications (CYC), Cape Town, South Africa, 22-27 September 2019.

9. Barnhart, T.E.; Engle, J.W.; Severin, G.W.; Valdovinos, H.F.; Gagnon, K.; Nickles, R.J. An after-market, five-port vertical beam line extension for the PETtrace. AIP Conf. Proc. 2012, 1509, 21-24. [CrossRef]

10. Hoehr, C.; Uittenbosch, T.; Verzilov, V.; English, W.; Buckley, K.; Gray, D.; Kellog, S.; Cameron, D.; Schaffer, P. A real-time beam-profile monitor for a PET cyclotron. AIP Conf. Proc. 2012, 1509, 41-45. [CrossRef]

11. Braccini, S.; Ereditato, A.; Scampoli, P.; Von Bremen, K. The New Bern Cyclotron Laboratory for Radioisotope Production and Research. In Proceedings of the IPAC2011, San Sebastian, Spain, 4-9 September 2011. Available online: www.jacow.org (accessed on 9 March 2021).

12. Braccini, S. The new Bern PET cyclotron, its research beam line, and the development of an innovative beam monitor detector. AIP Conf. Proc. 2013, 1525, 144-150.

13. Auger, M.; Braccini, S.; Ereditato, A.; Nesteruk, K.P.; Scampoli, P. Low current performance of the Bern medical cyclotron down to the pA range. Meas. Sci. Technol. 2015, 26, 094006. [CrossRef]

14. Dehnel, M.P.; Potkins, D.E.; Stewart, T.M. An Integrated Self-Supporting Mini-Beamline for PET Cyclotrons. In Proceedings of the International Conference on Cyclotrons and their Applications (CYC), Vancouver, BC, Canda, 16-20 September 2013.

15. Auger, M.; Braccini, S.; Carzaniga, T.S.; Ereditato, A.; Nesteruk, K.P.; Scampoli, P. A detector based on silica fibers for ion beam monitoring in a wide current range. J. Instrum. 2016, 11, P03027. [CrossRef] 
16. Potkins, D.E.; Braccini, S.; Nesteruk, K.P.; Carzaniga, T.S.; Vedda, A.; Chiodini, N.; Timmermans, J.; Melanson, S.; Dehnel, M.P. A Low-Cost Beam Profiler Based On Cerium-Doped Silica Fibres. In Proceedings of the CAARI 2016, Ft. Worth, TX, USA, 30 October-4 November 2016.

17. Belver-Aguilar, C.; Carzaniga, T.S.; Gsponer, A.; Häffner, P.; Scampoli, P.; Schmid, M.; Braccini, S.; Molinari, G. Development of Novel Non-Destructive 2D and 3D Beam Monitoring Detectors at the Bern Medical Cyclotron. In Proceedings of the IBIC2020, Santos, Brazil, 14-18 September 2020; ISBN 978-3-95450-222-6. [CrossRef]

18. Nesteruk, K.P.; Auger, M.; Braccini, S.; Carzaniga, T.S.; Ereditato, A.; Scampoli, P. A system for online beam emittance measurements and proton beam characterization. J. Instrum. 2018, 13, P01011. [CrossRef] 